\title{
New view of enteric nervous system development
}

Enteric neural crest cells (ENCCs) migrate during development to give rise to the enteric nervous system (ENS) in the mouse gut. Using time-lapse imaging analyses, Hideki Enomoto and colleagues have now started to chart this migration, providing new insights into the development of the ENS.

Mice were genetically engineered to express the photoconvertible protein KikumeGR in all ENCCs, thereby enabling study of the whole ENCC cellular population. KikumeGR changes its emission wavelength from green to red following UV irradiation; this colour change was visualised using fluorescence microscopy.

By analysing explant cultures of the gut taken from mouse embryos at different stages of development, the researchers discovered that 'a population of ENCCs traverse the mesentery from the midgut [which forms the small intestine] to the hindgut [which forms the large intestine] at a developmental period when these two gut regions are transiently juxtaposed', reports Enomoto. Interestingly, these so-called trans-mesenteric ENCCs seem to constitute a large proportion of the ENS in the colon.

'Our observation that a subset of ENCCs migrate across the mesentery revises the traditional view that the development of the ENS occurs by unidirectional, rostralto-caudal migration of ENCCs from the foregut to the caudal end of the hindgut,' he continues.

A failure of gut colonisation by ENCCs leads to Hirschsprung disease (intestinal aganglionosis). Using a mouse model of Hirschsprung disease, the researchers confirmed a delay in gut colonization by ENCCs and suggest that a defect in the migration of trans-mesenteric ENCCs causes this disease.

The investigators now plan to determine the molecular and cellular characteristics of trans-mesenteric ENCCs. 'Moreover,

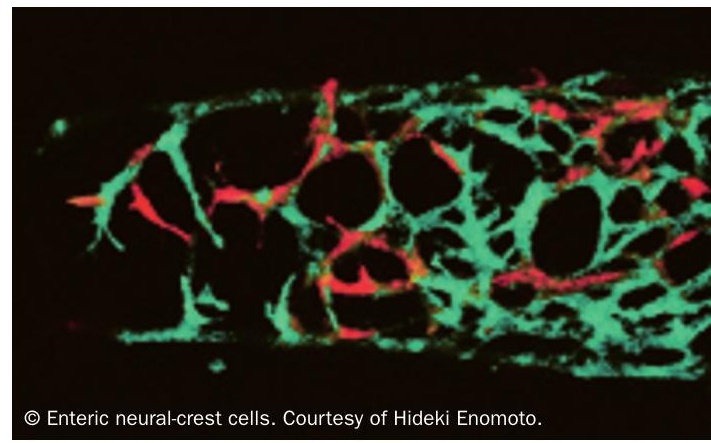

we aim to characterize the potential role of this population of ENCCs in the pathogenesis of Hirschsprung disease, which might expand our knowledge of the molecular mechanisms that underlie this disease,' says Enomoto.

\section{Katherine Smith}

Original article Nishiyama, C. et al. Trans-mesenteric neural crest cells are the principal source of the colonic enteric nervous sytem. Nat. Neurosci. doi:10.1038/nn.3184 